\title{
Analysis of Nonequilibrium Transport Properties of Interacting Quantum Wire Models
}

\author{
Yangdong Zheng \\ Research and Development Department, Mitsubishi Electric (China) Company Limited, Shanghai, China \\ Email: zhengyd09@163.com
}

How to cite this paper: Zheng, Y.D. (2019) Analysis of Nonequilibrium Transport Properties of Interacting Quantum Wire Models. Journal of Applied Mathematics and Physics, 7, 1677-1685.

https://doi.org/10.4236/jamp.2019.78114

Received: May 25, 2019

Accepted: August 9, 2019

Published: August 12, 2019

\begin{abstract}
We analyze nonequilibrium electronic transport properties of a typical interacting three-site quantum wire model within Hartree-Fock approximation making use of Keldysh formalism. Some rigorous formulas are provided for direct calculations when Coulomb repulsion is present. According to numerical calculations using above formulas, we investigate the conductance, transport currents, and on site electronic charges of the wire on some special occasions in the interacting case, and also compare them with the results in the noninteracting case.
\end{abstract}

\section{Keywords}

Quantum Wire Model, Nonequilibrium Transport Property, Conductance, Keldysh Formalism

\section{Introduction}

With advantage of top-down and bottom-up fabrication techniques for nanometer scale structures, it becomes possible to create quantum wire (QW) with the diameter of the order of the Fermi wavelength, and to experimentally study the quantum transport properties through them [1] [2]. In order to understand the experiment results, furthermore, to predict transport properties for applications in the future nanodevice design, theoretical approaches for clarifying QW electronic transport become necessary. In this paper, we present some theoretical formulas and numerical results for nonequilibrium electronic transport, using a simplest interacting model of three-site QW, in which each site has a single level. This method also could be extended to analyze the non- 
equilibrium transport properties of QWs containing much more sites with extended states.

In this study, the rigorous formulas of conductance, transport current and charge distributions for the three-site QW model are provided within Hartree-Fock approximation, based on the nonequilibrium transport theory (Keldysh formalism) [3] [4] [5]. From these formulas, the relevance between the transport properties and the temperature or the parameters in the Hamiltonian is expressed clearly and can be investigated in detail. In the noninteracting case $(U=0)$, we focus on the resonant tunneling transport and conductance quantization phenomenon. While in the interacting case $(U>0)$, we investigate Coulomb blockade and metal-insulator transition, as well as spin transport properties in the QW. It is reasonable to consider that the results of our study are available in the complicated case of actual QWs which are probably longer and thicker containing a larger number of atoms (sites) having multiple levels.

\section{Model and Formulation}

\subsection{Model}

We consider a one-dimensional QW with three lattice sites which are mutually coupled by tunneling barriers. They are combined with two external electrodes as shown in Figure 1. The tight-binding Hamiltonian of such system is described by Equation (1). Here $\hat{\boldsymbol{c}}_{k \sigma, \alpha}^{+}$and $\hat{\boldsymbol{c}}_{k \sigma, \alpha}(\alpha=\mathrm{L}$ or R) denote creation and annihilation operators of an electron with wave vector $k$ and spin $\sigma$ within $\mathrm{L}$ or $\mathrm{R}$ one-dimension perfect crystalline electrodes. The same operators of an electron within $i$-th site of the centre wire are denoted by $\hat{\boldsymbol{d}}_{i \sigma}^{+}$and $\hat{\boldsymbol{d}}_{i \sigma} \cdot \varepsilon_{k \sigma, \alpha}$ and $\varepsilon_{i \sigma}$ are on-site energies in the electrodes and wire region, respectively. The transfer integrals between the nearest-neighbor sites are $t_{i, j \sigma}$. The sites labelled by 1 and 3 are connected to the left and right electrode, respectively, and $V_{\alpha, k \sigma}$ denote the tunnel combination integrals between those boundary sites and the electrodes. The on-site Coulomb repulsion energies are denoted by $U_{i}$. When bias voltage $V$ is applied to the wire, it can be regarded as electrochemical potentials, $\mu_{\mathrm{L}}$ and $\mu_{\mathrm{R}}$, associate with the left and right electrode, respectively ( $\mathrm{eV}=\mu_{\mathrm{L}}-$ $\left.\mu_{\mathrm{R}}\right)$. We assume that the electrodes are electric reservoirs, the capacities of which are large enough that $\mu_{\mathrm{L}}$ and $\mu_{\mathrm{R}}$ are not perturbed by the transport current. In the case of $\mu_{\mathrm{L}}>\mu_{\mathrm{R}}$, electrons will flow from the left electrode to the right electrode.

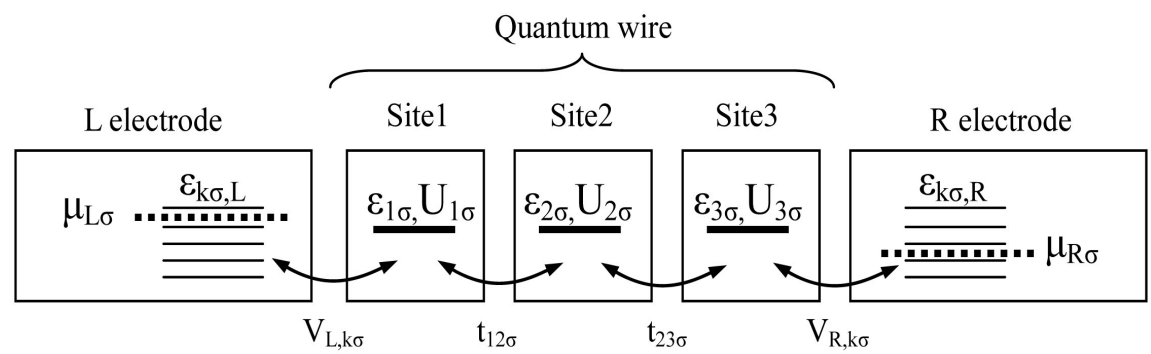

Figure 1. The model of three-site quantum wires combined with two external electrodes. 


$$
\begin{aligned}
& \hat{\boldsymbol{H}}=\sum_{k \sigma}\left(\varepsilon_{k \sigma, \mathrm{L}} \hat{\boldsymbol{c}}_{k \sigma, \mathrm{L}}^{+} \hat{\boldsymbol{c}}_{k \sigma, \mathrm{L}}+\varepsilon_{k \sigma, \mathrm{R}} \hat{\boldsymbol{c}}_{k \sigma, \mathrm{R}}^{+} \hat{\boldsymbol{c}}_{k \sigma, \mathrm{R}}\right)+\varepsilon_{1 \sigma} \hat{\boldsymbol{d}}_{1 \sigma}^{+} \hat{\boldsymbol{d}}_{1 \sigma}+\varepsilon_{2 \sigma} \hat{\boldsymbol{d}}_{2 \sigma}^{+} \hat{\boldsymbol{d}}_{2 \sigma} \\
& +\varepsilon_{3 \sigma} \hat{\boldsymbol{d}}_{3 \sigma}^{+} \hat{\boldsymbol{d}}_{3 \sigma}+\sum_{\sigma}\left(t_{12 \sigma} \hat{\boldsymbol{d}}_{1 \sigma}^{+} \hat{\boldsymbol{d}}_{2 \sigma}+t_{12 \sigma}^{*} \hat{\boldsymbol{d}}_{2 \sigma}^{+} \hat{\boldsymbol{d}}_{1 \sigma}+t_{23 \sigma} \hat{\boldsymbol{d}}_{2 \sigma}^{+} \hat{\boldsymbol{d}}_{3 \sigma}+t_{23 \sigma}^{*} \hat{\boldsymbol{d}}_{3 \sigma}^{+} \hat{\boldsymbol{d}}_{2 \sigma}\right) \\
& +U_{1} \hat{\boldsymbol{d}}_{1 \uparrow}^{+} \hat{\boldsymbol{d}}_{1 \uparrow} \hat{\boldsymbol{d}}_{1 \downarrow}^{+} \hat{\boldsymbol{d}}_{1 \downarrow}+U_{2} \hat{\boldsymbol{d}}_{2 \uparrow}^{+} \hat{\boldsymbol{d}}_{2 \uparrow} \hat{\boldsymbol{d}}_{2 \downarrow}^{+} \hat{\boldsymbol{d}}_{2 \downarrow}+U_{3} \hat{\boldsymbol{d}}_{3 \uparrow}^{+} \hat{\boldsymbol{d}}_{3 \uparrow} \hat{\boldsymbol{d}}_{3 \downarrow}^{+} \hat{\boldsymbol{d}}_{3 \downarrow} \\
& +\sum_{k \sigma}\left(V_{\mathrm{L}, k \sigma} \hat{\boldsymbol{c}}_{k \sigma, \mathrm{L}}^{+} \hat{\boldsymbol{d}}_{1 \sigma}+V_{\mathrm{L}, k \sigma}^{*} \hat{\boldsymbol{d}}_{1 \sigma}^{+} \hat{\boldsymbol{c}}_{k \sigma, \mathrm{L}}\right)+\sum_{k \sigma}\left(V_{\mathrm{R}, k \sigma} \hat{\boldsymbol{c}}_{k \sigma, \mathrm{R}}^{+} \hat{\boldsymbol{d}}_{3 \sigma}+V_{\mathrm{R}, k \sigma}^{*} \hat{\boldsymbol{d}}_{3 \sigma}^{+} \hat{\boldsymbol{c}}_{k \sigma, \mathrm{R}}\right)
\end{aligned}
$$

\subsection{Formulation}

We consider a system consisting of three regions, a left electrode, a right electrode and a intermediate wire, they uncouple and each one maintains its noninteracting thermal equilibrium when $t=-\infty$, then turn on the perturbation coupling between the wire and electrodes adiabatically with a route of $t=-\infty \rightarrow 0 \rightarrow+\infty \rightarrow-\infty$ (Keldysh contour). According to the quantum statistical theory (perturbation expansion), any nonequilibrium observable physical quantity at time $t$ can be expressed exactly by Keldysh Green's Functions (GF), such as correlation function $G_{i j}^{<}\left(t, t_{0}\right)=\mathrm{i}\left\langle\hat{\boldsymbol{c}}_{i}^{+}(t) \hat{\boldsymbol{c}}_{j}\left(t_{0}\right)\right\rangle$ and retarded/advanced GF $G_{i j}^{\mathrm{r}(\mathrm{a})}\left(t, t_{0}\right)=\mp \mathrm{i} \theta\left[ \pm\left(t-t_{0}\right)\right]\left\langle\hat{\boldsymbol{c}}_{i}(t) \hat{\boldsymbol{c}}_{j}^{+}\left(t_{0}\right)+\hat{\boldsymbol{c}}_{j}^{+}\left(t_{0}\right) \hat{\boldsymbol{c}}_{i}(t)\right\rangle \quad$ (Keldysh formalism) [3] [4]. These GFs can be solved from Dyson-equation.

The self-energies resulting from wire-electrode coupling and Coulomb repulsion are derived within Hartree-Fock approximation and are shown in Equation (2) and Equation (3), respectively:

$$
\begin{gathered}
{\left[\begin{array}{cc}
\Sigma_{p q}^{(t)--}(\varepsilon) & \Sigma_{p q}^{(t)<}(\varepsilon) \\
\sum_{p q}^{(t)>}(\varepsilon) & \Sigma_{p q}^{(t)++}(\varepsilon)
\end{array}\right]} \\
=\sum_{k \sigma} V_{\mathrm{L}, k \sigma}^{*} \delta_{p, 1 \sigma} \delta_{q, k \sigma-\mathrm{L}}\left[\begin{array}{cc}
1 & 0 \\
0 & -1
\end{array}\right]+\sum_{k \sigma} V_{\mathrm{L}, k \sigma} \delta_{p, k \sigma-\mathrm{L}} \delta_{q, 1 \sigma}\left[\begin{array}{cc}
1 & 0 \\
0 & -1
\end{array}\right] \\
+\sum_{k \sigma} V_{\mathrm{R}, k \sigma}^{*} \delta_{p, N \sigma} \delta_{q, k \sigma-\mathrm{R}}\left[\begin{array}{cc}
1 & 0 \\
0 & -1
\end{array}\right]+\sum_{k \sigma} V_{\mathrm{R}, k \sigma} \delta_{p, k \sigma-\mathrm{R}} \delta_{q, N \sigma}\left[\begin{array}{cc}
1 & 0 \\
0 & -1
\end{array}\right] \\
\sum_{p q}^{(t)--}(\varepsilon)=\sum_{k \sigma} V_{\mathrm{L}, k \sigma}^{*} \delta_{p, 1 \sigma} \delta_{q, k \sigma-\mathrm{L}}+\sum_{k \sigma} V_{\mathrm{L}, k \sigma} \delta_{p, k \sigma-\mathrm{L}} \delta_{q, 1 \sigma} \\
+\sum_{k \sigma} V_{\mathrm{R}, k \sigma}^{*} \delta_{p, N \sigma} \delta_{q, k \sigma-\mathrm{R}}+\sum_{k \sigma} V_{\mathrm{R}, k \sigma} \delta_{p, k \sigma-\mathrm{R}} \delta_{q, N \sigma} \\
\sum_{p q}^{(t)++}(\varepsilon)=-\sum_{k \sigma} V_{\mathrm{L}, k \sigma}^{*} \delta_{p, 1 \sigma} \delta_{q, k \sigma-\mathrm{L}}-\sum_{k \sigma} V_{\mathrm{L}, k \sigma} \delta_{p, k \sigma-\mathrm{L}} \delta_{q, 1 \sigma}, \\
-\sum_{k \sigma} V_{\mathrm{R}, k \sigma}^{*} \delta_{p, N \sigma} \delta_{q, k \sigma-\mathrm{R}}-\sum_{k \sigma} V_{\mathrm{R}, k \sigma} \delta_{p, k \sigma-\mathrm{R}} \delta_{q, N \sigma} \\
\sum_{p q}^{(t)<}(\varepsilon)=0, \quad \sum_{p q}^{(t)>}(\varepsilon)=0 \\
\sum_{n_{1} \sigma, n_{2} \sigma}^{(i)-}(\varepsilon)=U_{n \bar{\sigma}} \rho_{n \bar{\sigma}, n \bar{\sigma}}(\varepsilon) / e, \quad \sum_{p q}^{(i)>}(\varepsilon)=0, \\
\sum_{n_{1} \sigma, n_{2} \sigma^{\prime}}^{(i)+}(\varepsilon)=\left(-U_{n \bar{\sigma}}\right) \rho_{n \bar{\sigma}, n \bar{\sigma}}(\varepsilon) / e, \quad \sum_{p q}^{(i)<}(\varepsilon)=0
\end{gathered}
$$

Corresponding retarded/advanced self-energies are given by

$$
\mathrm{i} \Gamma_{\alpha \sigma}^{\mathrm{r}}(\varepsilon)=-\mathrm{i} \pi v_{\alpha, \sigma}(\varepsilon)\left|V_{\alpha, k \sigma}\right|^{2}, \quad \mathrm{i} \Gamma_{\alpha \sigma}^{\mathrm{a}}(\varepsilon)=\mathrm{i} \pi v_{\alpha, \sigma}(\varepsilon)\left|V_{\alpha, k \sigma}\right|^{2}
$$

where $v_{\alpha, \sigma}(\varepsilon)$ is the density-of-states (DOS) in the electrodes, $\alpha=\mathrm{L}, \mathrm{R}$. 
The single spin current flowing in the wire and the spin electron charge on the site $\mathrm{n}$ are given by Equation (5) and Equation (6), respectively [5]:

$$
\begin{gathered}
I_{\sigma}=\frac{e}{\hbar} \sum_{k \sigma, n} \int_{-\infty}^{+\infty} \frac{\mathrm{d} \varepsilon}{2 \pi}\left[V_{k \sigma-\mathrm{L}, n \sigma} G_{n, k \sigma-\mathrm{L}}^{<}(\varepsilon)-V_{k \sigma-\mathrm{L}, n \sigma}^{*} G_{k \sigma-\mathrm{L}, n}^{<}(\varepsilon)\right] \\
\rho_{n \sigma}=e(-\mathrm{i}) \frac{1}{2 \pi} \int_{-\infty}^{+\infty} \mathrm{d} \varepsilon G_{n \sigma, n \sigma}^{<}(\varepsilon)
\end{gathered}
$$

From Equation (5) and Equation (6), the following transport formulas can be obtained by correlation functions calculations straightforwardly $\left(f_{\mu \sigma}\right.$ is the Fermi distribution function).

The spin current is

$$
J_{\sigma}\left(\mu_{\mathrm{L} \sigma}, \mu_{\mathrm{R} \sigma}\right)=\frac{e}{h} \int_{-\infty}^{+\infty} \mathrm{d} \varepsilon\left(f_{\mu_{\mathrm{L} \sigma}}-f_{\mu_{\mathrm{R}} \sigma}\right) \frac{4 \Gamma_{\mathrm{L} \sigma}^{\mathrm{r}} \Gamma_{\mathrm{R} \sigma}^{\mathrm{r}}\left|t_{12 \sigma} t_{23 \sigma}\right|^{2}}{\left|B_{\sigma}^{\mathrm{r}}\right|^{2}}
$$

the spin conductance is

$$
G_{\sigma}\left(\mu_{\mathrm{L}(\mathrm{R}) \sigma}\right)=\frac{e^{2}}{h} \int_{-\infty}^{+\infty} \mathrm{d} \varepsilon\left\{\frac{4 \Gamma_{\mathrm{L} \sigma}^{\mathrm{r}} \Gamma_{\mathrm{R} \sigma}^{\mathrm{r}}\left|t_{12 \sigma} t_{23 \sigma}\right|^{2}}{\left|B_{\sigma}^{\mathrm{r}}\right|^{2}}\left(\frac{1}{4 k_{\mathrm{B}} T} \frac{1}{\cosh ^{2}\left(\frac{\varepsilon-\mu_{\mathrm{L}(\mathrm{R}) \sigma}}{2 k_{\mathrm{B}} T}\right)}\right)\right\}
$$

and the up-spin electron charges on each site of the wire are expressed by

$\rho_{1 \uparrow, 1 \uparrow}\left(\mu_{\mathrm{L} \uparrow}, \mu_{\mathrm{R} \uparrow}\right)$

$=\frac{e}{\pi} \int_{-\infty}^{+\infty} \mathrm{d} \varepsilon\left\{\frac{f_{\mu_{\mathrm{L}} \uparrow} \Gamma_{\mathrm{L} \uparrow}^{\mathrm{r}}\left[\left(\left(x_{2 \uparrow}-U_{2} \rho_{2 \downarrow, 2 \downarrow} / e\right)\left(x_{3 \uparrow}-U_{3} \rho_{3 \downarrow, 3 \downarrow} / e\right)-\left|t_{23 \uparrow}\right|^{2}\right)^{2}+\left(\Gamma_{\mathrm{R} \uparrow}^{\mathrm{r}}\right)^{2}\left(x_{2 \uparrow}-U_{2} \rho_{2 \downarrow, 2 \downarrow} / e\right)^{2}\right]+f_{\mu_{\mathrm{R}} \uparrow} \Gamma_{\mathrm{R} \uparrow}^{\mathrm{r} \uparrow}\left|t_{12 \uparrow} t_{23 \uparrow}\right|^{2}}{\left|B_{\uparrow}^{\mathrm{r}}\right|^{2}}\right\}$

$\rho_{2 \uparrow, 2 \uparrow}\left(\mu_{\mathrm{L} \uparrow}, \mu_{\mathrm{R} \uparrow}\right)$

$=\frac{e}{\pi} \int_{-\infty}^{+\infty} \mathrm{d} \varepsilon\left\{\frac{f_{\mu_{\mathrm{L}} \uparrow} \Gamma_{\mathrm{L} \uparrow}^{\mathrm{r}}\left[\left(x_{3 \uparrow}-U_{3} \rho_{3 \downarrow, 3 \downarrow} / e\right)^{2}+\left(\Gamma_{\mathrm{R} \uparrow}^{\mathrm{r}}\right)^{2}\right]\left|t_{12 \uparrow}\right|^{2}+f_{\mu_{\mathrm{R}} \uparrow} \Gamma_{\mathrm{R} \uparrow}^{\mathrm{r}}\left[\left(x_{1 \uparrow}-U_{1} \rho_{1 \downarrow, 1 \downarrow} / e\right)^{2}+\left(\Gamma_{\mathrm{L} \uparrow}^{\mathrm{r}}\right)^{2}\right]\left|t_{23 \uparrow}\right|^{2}}{\left|B_{\uparrow}^{\mathrm{r}}\right|^{2}}\right\}$

$\rho_{3 \uparrow, 3 \uparrow}\left(\mu_{\mathrm{L} \uparrow}, \mu_{\mathrm{R} \uparrow}\right)$

$=\frac{e}{\pi} \int_{-\infty}^{+\infty} \mathrm{d} \varepsilon\left\{\frac{f_{\mu_{\mathrm{R}} \uparrow} \Gamma_{\mathrm{R} \uparrow}^{\mathrm{r}}\left|t_{12 \uparrow} t_{23 \uparrow}\right|^{2}+f_{\mu_{\mathrm{R}} \uparrow} \Gamma_{\mathrm{R} \uparrow}^{\mathrm{r}}\left[\left(\left(x_{1 \uparrow}-U_{1} \rho_{1 \downarrow, 1 \downarrow} / e\right)\left(x_{2 \uparrow}-U_{2} \rho_{2 \downarrow, 2 \downarrow} / e\right)-\left|t_{1 \uparrow \uparrow}\right|^{2}\right)^{2}+\left(\Gamma_{\mathrm{L} \uparrow}^{\mathrm{r}}\right)^{2}\left(x_{2 \uparrow}-U_{2} \rho_{2 \downarrow, 2 \downarrow} / e\right)^{2}\right]}{\left|B_{\uparrow}^{\mathrm{r}}\right|^{2}}\right\}$

where $x_{1 \sigma}=\varepsilon-\varepsilon_{1 \sigma}, x_{2 \sigma}=\varepsilon-\varepsilon_{2 \sigma}, x_{3 \sigma}=\varepsilon-\varepsilon_{3 \sigma}$. Value $B$ is given by

$$
\begin{aligned}
& B_{\uparrow}^{\mathrm{r}}=\left[\varepsilon-\left(\varepsilon_{1 \uparrow}+U_{1} \rho_{1 \downarrow, 1 \downarrow} / e\right)+\mathrm{i} \Gamma_{\mathrm{L} \uparrow}^{\mathrm{r}}\right]\left[\varepsilon-\left(\varepsilon_{2 \uparrow}+U_{2} \rho_{2 \downarrow, 2 \downarrow} / e\right)\right] \\
& \times\left[\varepsilon-\left(\varepsilon_{3 \uparrow}+U_{3} \rho_{3 \downarrow, 3 \downarrow} / e\right)+\mathrm{i} \Gamma_{\mathrm{R} \uparrow}^{\mathrm{r}}\right] \\
& -\left[\varepsilon-\left(\varepsilon_{1 \uparrow}+U_{1} \rho_{1 \downarrow, 1 \downarrow} / e\right)+\mathrm{i} \Gamma_{\mathrm{L} \uparrow}^{\mathrm{r}}\right]\left|t_{23 \uparrow}\right|^{2} \\
& -\left[\varepsilon-\left(\varepsilon_{3 \uparrow}+U_{3} \rho_{3 \downarrow, 3 \downarrow} / e\right)+\mathrm{i} \Gamma_{\mathrm{R} \uparrow}^{\mathrm{r}}\right]\left|t_{12 \uparrow}\right|^{2}
\end{aligned}
$$


The electron charge formulas for down-spin can be obtained by exchanging the subscript $\uparrow$ and $\downarrow$ in the up-spin formulas above.

\section{Numerical Results and Interpretations}

In this section, we calculate the transport properties of the three-site QW in some special cases applying the formulas in previous section. We assume that $\varepsilon_{i \sigma}=0, \quad t_{12 \uparrow \downarrow}=t_{23 \uparrow \downarrow}=t, \quad V_{\mathrm{L}, k \uparrow \downarrow}=V_{\mathrm{R}, k \uparrow \downarrow}=V, \quad \Gamma_{\mathrm{L} \uparrow \downarrow}(\varepsilon)=\Gamma_{\mathrm{R} \uparrow \downarrow}(\varepsilon)=\Gamma \quad$ and $U_{1}=U_{2}=U_{3}=U$ All of the energies are normalized by the transfer integral $t$. Especially, the normalized self-energy is defined as $\gamma=\Gamma / t$ in the following numerical calculations.

\subsection{Noninteracting Case $(U=0)$}

The numerical results of conductance, current and electron charges in the three sites of the wire as a function of electrochemical potential $\mu$ for several values of $\gamma$ are illustrated in Figures 2-4.

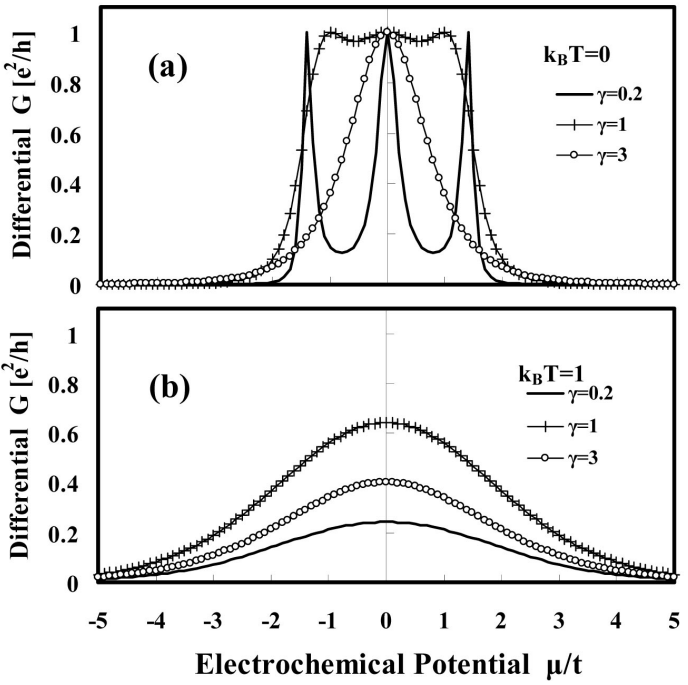

Figure 2. Conductance as a function of electrochemical potential $\mu$ for self-energy $\gamma=0.2$, 1 and 3 (normalized by transfer energy $t$ ). (a) When $k_{\mathrm{B}} T=0$ ( $\left.T=0 \mathrm{~K}\right)$; (b) When $k_{\mathrm{B}} T=$ $1(T>0 \mathrm{~K})$.

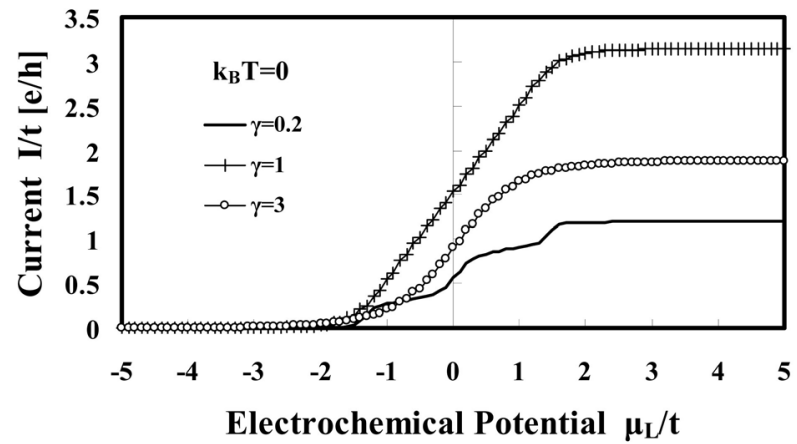

Figure 3. Transport current as a function of electrochemical potential of left electrode $\mu_{\mathrm{L}}$ $\left(\mu_{\mathrm{R}}=-5\right)$ for self-energy $\gamma=0.2,1$ and 3 , when $T=0 \mathrm{~K}$. 


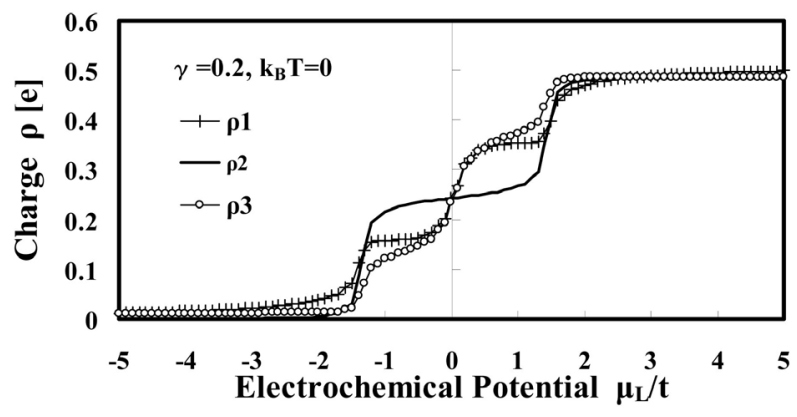

Figure 4. Electronic charges in the three sites as a function of electrochemical potential of left electrode $\mu_{\mathrm{L}}\left(\mu_{\mathrm{R}}=-5\right)$ for self-energy $\gamma=0.2$, when $T=0 \mathrm{~K}$.

The behavior of conductance and transport current changes dramatically when the value of $\gamma$ crosses unity. When $\gamma<1$, the conductance has three maximums at $\mu / t=0$ and $\mu / t= \pm \sqrt{2}$, and the corresponding current increases intermittently with a step shape. These phenomena imply that resonant tunneling and conductance quantization take place easily in this case. Whereas when $\gamma \geq 1$, these quantum effects in transport will disappear gradually with the increase of $\gamma$. In the case of $T>0 \mathrm{~K}$, the line shapes of the transport characteristics become not to change so much and become all smoother than those in $T=0 \mathrm{~K}$ due to the thermal fluctuations. The charges distributions shown in Figure 4 results in the fact that in the area of $\mu_{\mathrm{L}}<0$, a minus charge barrier will be formed at the boundary of the wire, whereas in the area of $\mu_{\mathrm{L}}>0$, a plus charge barrier will be formed.

\subsection{Interacting Case $(U>0)$}

We select comparative small value of $U(U<5)$ to investigate Coulomb interaction effects in transport due to the limits of Hartree-Fock approximation. The transport properties are computed by self-consistent calculations concern with site charges $\rho_{n} \uparrow$. The initial site charges are decided by the ground state of the three-site QW with half-filling $(N=3)$ assumption, which is an antiferromagnet state with total spin of $+1 / 2$.

The numerical results of spin conductance in the case of $\gamma=0.2$ and 1 as a function of $\mu$ for several values of $U$ are illustrated in Figure 5(1) and Figure 5(2), respectively. Compared with the case of $U=0$, the conductance curves shift to right and peaks are broadened with the increase of $U$. When $U>2 \gamma$, the peaks of conductance start to split into two corresponding to up or down spin conductance. These phenomena all result from the changes of spin orbits in the wire due to the Coulomb repulsion between the up and down spin electrons on sites. The series of peaks and valleys in the conductance characteristics can be considered a synthetic effect of resonant tunneling and Coulomb blockade. One of the valley happen to shift on the Fermi-level of the wire $(\mu=0)$, the metal-insulator transition (Mott transition) will takes place. We show the spin conductance as a function of $U$ for several values of $\gamma$ when $\mu=0$ in Figure 6. This result indicates that, if the self-energy $\gamma$ has small value compared with 

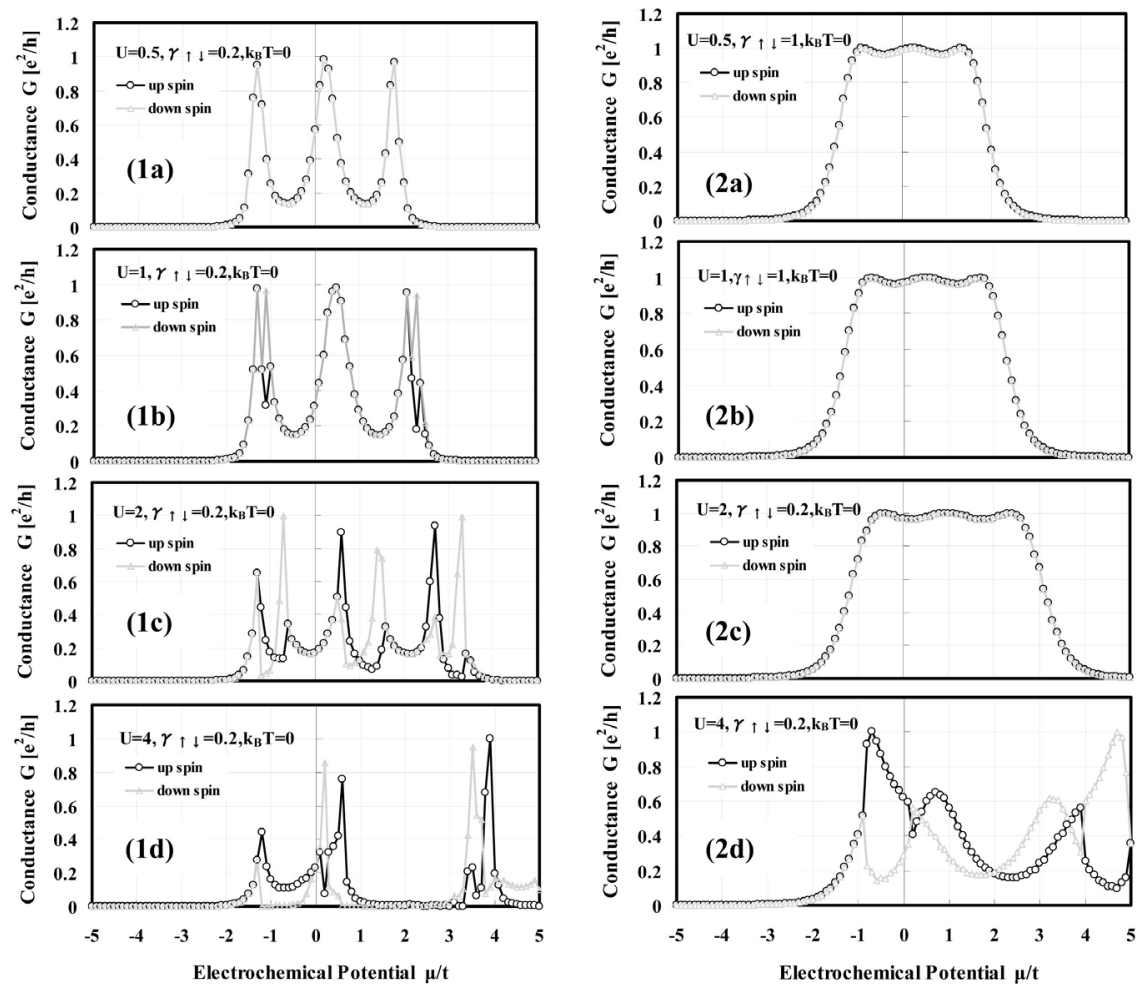

Figure 5. Up-spin and down-spin conductance as a function of electrochemical potential $\mu$ for self-energy $\gamma=0.2$ and $\gamma=1$, when $T=0 \mathrm{~K}$. (1a)-(1d) $\gamma=0.2$, (2a)-(2d) $\gamma=1$, with different on-site Coulomb repulsion energies(a) $U=0.5$; (b) $U=1$; (c) $U=2$; (d) $U=4$.

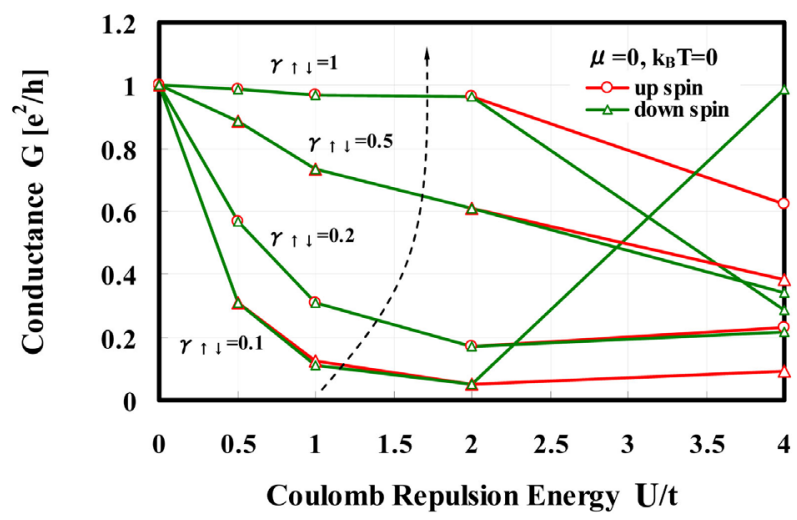

Figure 6. Up-spin and down-spin conductance as a function of $U$ for self-energy $\gamma=0.1$, $0.2,0.5$ and 1 , when electrochemical potential $\mu=0$ and $T=0 \mathrm{~K}$.

$U$, generally the spin conductance will rapidly decrease with the increase of $U$, and the wire becomes an insulator from a metal (Mott transition).

We illustrate the spin current as a function of left electrode potential $\mu_{\mathrm{L}}\left(\mu_{\mathrm{R}}=\right.$ -5 ) for several values of $U$ in Figure 7(1) when $\gamma=0.2$. Because the self-energy $\gamma$ has a small value, the nonequilibrium spin current gradually decreases with the increase of $U$. Meanwhile, the line shape of the up spin current separates from that of the down spin current.

In Figure 7(2), we demonstrate the up and down spin current as a function 

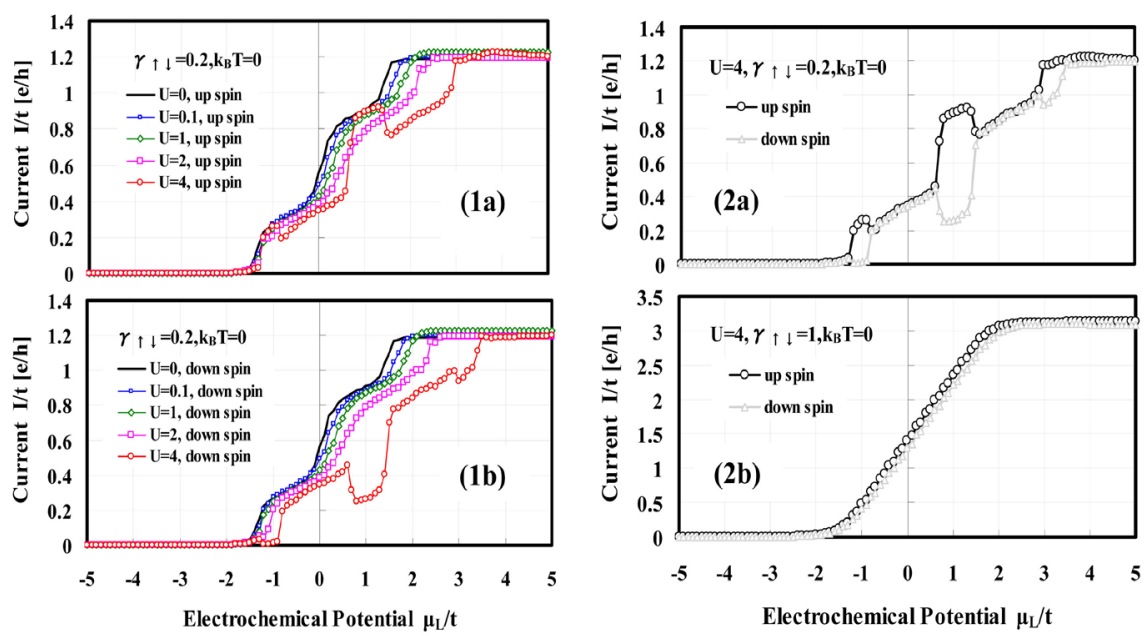

Figure 7. (1) Spin transport current as a function of electrochemical potential of left electrode $\mu_{\mathrm{L}}\left(\mu_{\mathrm{R}}=-5\right)$ for $U=0,0.5,1,2$, and 4 , when self-energies $\gamma=0.2$ and $T=0 \mathrm{~K}$. (1a) Up-spin. (1b) Down-spin. (1) Up-spin and down-spin transport current as a function of electrochemical potential of left electrode $\mu_{\mathrm{L}}\left(\mu_{\mathrm{R}}=-5\right)$ for $U=4$, when $\mathrm{T}=0 \mathrm{~K}$. (2a) $\gamma=$ 0.2. (2b) $\gamma=1$.

of $\mu_{\mathrm{L}}\left(\mu_{\mathrm{R}}=-5\right)$ for $\gamma=0.2$ and 1 when $U$ has a large value of 4 . Especially, in the case of $\gamma=0.2$, when $\mu_{\mathrm{L}}$ approaches some positions where the conductance has peak values in Figure 5(1), the up-spin current increases while the down-spin current decreases, indicating that the spin polarization takes place in the wire.

\section{Summary}

Based on the Keldysh formalism, we provided some rigorous formulas of nonequilibrium electronic transport for a typical interacting three-site QW model within Hartree-Fock approximation when Coulomb repulsion is present. According to numerical calculations, we investigated the conductance, transport current and electronic charge distribution of the three-site QW in some special occasions. In the noninteracting case, when self-energy $\gamma<0$, the resonant tunneling transport and the conductance quantization can be easily observed. The transport properties of up-spin are identical with those of down-spin. While if the Coulomb interaction is present, the conductance curves shift to right and the peaks are broadened with the increase of $U$ because of electron-electron repulsions. When $U>2 \gamma$, the peaks of conductance split into two. The Coulomb blockade and metal-insulator transition (Mott transition) phenomena are obvious if $\gamma$ has a small value compared with $U$. The conductance and transport current of the up-spin also become quite different from those of the down-spin indicating that the spin polarization takes place in the wire.

\section{Acknowledgements}

This research was supported in part by Grants-in-Aid for the basic research and development of Mitsubishi Electric (China) Company Limited. 


\section{Conflicts of Interest}

The author declares no conflicts of interest regarding the publication of this paper.

\section{References}

[1] Iijima, S. and Ichihashi, T. (1993) Single-Shell Carbon Nanotubes of 1-nm Diameter. Nature (London), 363, 603. https://doi.org/10.1038/363603a0

[2] Ma, D.D.D., Lee, C.S., Au, F.C.K., Tong, S.Y. and Lee S.T. (2003) Small-Diameter Silicon Nanowire Surfaces. Science, 299, 1874.

https://doi.org/10.1126/science.1080313

[3] Keldysh, L.V. (1965) Sov. Phys. JETP, 20, 1018.

[4] Caroli, C., Combescot, R., Nozieres, P. and Saint-James, D. (1971) Phys. C, 4, 916. https://doi.org/10.1088/0022-3719/4/8/018

[5] Meir, Y. and Wingreen, N.S. (1992) Landauer Formula for the Current through an Interaction Electron Region. Phys. Rev. Lett, 68, 2512.

https://doi.org/10.1103/PhysRevLett.68.2512 